\title{
Apolipoprotein E genotyping using PCR-GoldMag lateral flow assay and its clinical applications
}

\author{
TING LIAN $^{1 *}$, WENLI HUI $^{1 *}$, XIANYING LI ${ }^{1}$, CHAO ZHANG $^{1}$, \\ JUANLI ZHU ${ }^{1}$, RUI LI ${ }^{2}$, YINSHENG WAN ${ }^{3}$ and YALI CUI ${ }^{1}$ \\ ${ }^{1}$ College of Life Sciences, Northwest University, Xi'an, Shaanxi 710069; \\ ${ }^{2}$ Department of Neurology, Shaanxi Provincial People's Hospital, Xi'an, Shaanxi 710068, P.R. China; \\ ${ }^{3}$ Department of Biology, Providence College, Providence, RI 02918, USA
}

Received February 12, 2016; Accepted August 3, 2016

DOI: $10.3892 / \mathrm{mmr} .2016 .5768$

\begin{abstract}
A polymerase chain reaction-gold magnetic nanoparticles lateral flow assay (PCR-GoldMag LFA) has been developed via integrating multiplex amplification refractory mutation system PCR (multi-ARMS-PCR) with GoldMag-based LFA for the visual detection of single-nucleotide polymorphisms (SNPs). This assay was applied to genotype Apolipoprotein E (ApoE). ApoE genotyping is important due to the predictive value for the development of coronary artery disease and Alzheimer's disease. The method requires two steps: i) Simultaneous amplifications of the two polymorphic codons (ApoE 158 and 112), performed in separated reactions using multi-ARMS-PCR; and ii) detection of the wild-type and mutant PCR products via dual immunoreactions, which can be performed in $\sim 5 \mathrm{~min}$. Within two LFAs, anti-digoxin antibody-conjugated GoldMag probes bind digoxin-labeled wild-type PCR products, and anti-fluorescein isothiocyanate (FITC) antibody-conjugated GoldMag probes bind FITC-labeled mutant PCR products. All PCR products are biotin labeled and are detected by streptavidin-coated regions on the LFA strip, resulting in a red color. The current approach is capable of detecting the SNPs of ApoE in $\sim 1.5 \mathrm{~h}$, with a broad detection range from 10-1,000 ng of genomic DNA. Thus, the present protocol may facilitate simple, fast and cost-effective screening for important SNPs, as demonstrated
\end{abstract}

Correspondence to: Professor Yali Cui, College of Life Sciences, Northwest University, 229 North Taibai Road, Xi'an, Shaanxi 710069, P.R. China

E-mail: yalicui@nwu.edu.cn

Professor Yinsheng Wan, Department of Biology, Providence College, 1 Cunningham Square, Providence, RI 02918, USA

E-mail: yswan@providence.edu

"Contributed equally

Key words: ApoE, multiplex amplification refractory mutation system PCR, lateral flow assay, polyelectrolyte, GoldMag nanoparticles by the evaluation of the prevalence of ApoE variants in a Han Chinese cohort.

\section{Introduction}

Increasing evidence has suggested that single-nucleotide polymorphisms (SNPs) may become a new generation of genetic markers and valuable indicators for clinical diagnosis and prognosis $(1,2)$. A polymorphism in Apolipoprotein E (ApoE) is one of the most widely studied polymorphisms, and has been considered to be a pre-symptomatic risk predictor for a variety of diseases, including coronary artery disease and late-onset Alzheimer's disease $(3,4)$. The molecular bases of ApoE polymorphism are cysteine (TGC)-arginine (CGC) interchanges at the one or both of residues 158 and 112 that determine three major alleles, designated E2, E3 and E4. This polymorphism leads to the presence of six genotypes in the general population: E2/E2, E3/E3, E4/E4, E2/E3, E2/E4 and E3/E4 (5).

Several methods have been established for identifying ApoE polymorphisms. These methods can be divided into two groups: i) Proteomic analyses using isoelectric focusing (6) or immunoassay reagents combined with mass spectrometry (7); and ii) genotyping techniques that detect sequence differences in the ApoE alleles, including polymerase chain reaction $(\mathrm{PCR})$ restriction fragment length polymorphism analysis (8), quantitative PCR analysis (9), mass spectrometry (10), amplification-refractory mutation system (11), denaturing high-performance liquid chromatography (12), TaqMan assays (13) and single base extension genotyping technology (14). Currently, ARMS is generally considered as a simple and cost-effective method. ARMS for ApoE genotyping, as previously described (11), requires four separate PCR reactions. The present study aimed to reduce the complexity of ApoE genotyping by combining the different primers into two PCR reactions. However, the method described still requires analysis by agarose gels, thus, limiting its clinical applications.

Accurate and rapid methods for ApoE genotyping are in ever-increasing demand. Nanoparticle-associated lateral flow assays (LFAs) have attracted considerable research interest, as they provide a promising approach to enable point-of-care nucleic acid detection (15). In the emerging 
and revolutionary diagnostic arena, $\mathrm{Fe}_{3} \mathrm{O}_{4} / \mathrm{Au}$ nanoparticles, formed of an iron oxide core with gold coating, are often used to improve the sensitivity of the immunosensor due to their stability, high surface-to-volume ratio and biocompatibility $(16,17)$. Polystyrenesulfonate (PSS) modification has been demonstrated to be an effective strategy to increase gold nanorods stability and compatibility for biological interactions (18). Therefore, the polyelectrolyte-coated gold magnetic (GoldMag) nanoparticle (PGMN)-mediated conjugates may be a colloidal, monodispersed particle probe to accurately detect a target with high sensitivity and specificity, based on LFAs in clinical diagnostics. A GoldMag based lateral-flow immunoassay was previously reported to rapidly, specifically and accurately analyze Treponema pallidum antibody, at the laboratory and clinical level (19). A recent study by our laboratory proposed a novel approach for the visual detection of MTHFR C677T polymorphisms via integrating the ARMS-PCR with GoldMag-based LFA (20); the assay involves two complementary PCR reactions for each SNP.

The current study describes a PCR-GoldMag LFA for ApoE genotyping based on the multi-ARMS-PCR and GoldMag LFA, and analyzes the distribution of ApoE variants in a Han Chinese cohort. It takes only two multi-ARMS-PCR reactions, using GoldMag-based LFAs, to distinguish directly the six different ApoE genotypes. The PCR-GoldMag LFA, as a simple and rapid method, enables visual identification of SNPs, and avoids complex steps, including pipetting, incubation, washing and data analysis. This novel method can also be easily extended to detect SNPs of other disease-associated genes.

\section{Materials and methods}

Materials and reagents. GoldMag nanoparticles $(5 \mathrm{mg} / \mathrm{ml})$ with a nanoflower structure (21) and lateral flow strips were provided by Xi'an GoldMag Nanobiotech Co., Ltd. (Xi'an, China). Buffers were prepared according to standard laboratory procedures. Water $(18.2 \mathrm{M} \Omega \mathrm{cm})$ purified by the Barnstead Nanopure Water System (Thermo Fisher Scientific, Inc., Waltham, MA, USA) was used for all sample preparation. All chemicals listed below were of analytical grade. Cetyltrimethylammonium bromide (CTAB; 99\% purity), dimethyl sulfoxide (DMSO) and PSS sodium salt (molecular weight, $70 \mathrm{kDa}$ ) were obtained from Sigma-Aldrich (Merck Millipore, Darmstadt, Germany). A mouse anti-digoxin antibody (catalog no. MAD53-310C) was purchased from Meridian Life Science, Inc. (Cincinnati, OH, USA). An anti-fluorescein isothiocyanate (FITC) antibody (catalog no. bs-0366R) and a goat anti-mouse IgG antibody (catalog no. bs-0296 G) were obtained from Beijing Biosynthesis Biotechnology Co., Ltd. (Beijing, China). Streptavidin was obtained from Promega Corporation (Madison, WI, USA). All labeled oligonucleotides were synthesized by Invitrogen (Thermo Fisher Scientific, Inc.). The sequences of each oligonucleotide are listed in Table I. HotMaster Taq DNA Polymerase kit was purchased from Tiangen Biotech Co., Ltd. (Beijing, China), and dNTP and uracil-DNA glycosylase (UDG) polymerase were from ShineGene Bio-Technologies, Inc. (Shanghai, China). The Lowry protein assay kit, bovine serum albumin (BSA) and calf serum were from Sigma-Aldrich (Merck Millipore).
Instruments. The Fourier transform-infrared (FT-IR) spectrum of particles was recorded using a Nicolet 5700 FT-IR spectrometer (Thermo Fisher Scientific, Inc.), followed by drying. A Hitachi H-600 transmission electron microscope (TEM; Hitachi, Ltd., Tokyo, Japan) was used to acquire images of particles, whereas particles size and zeta potential were characterized by dynamic light scattering using Zetasizer Nano ZS (Malvern Instruments Ltd., Malvern, UK). A 2550 UV-visible spectrophotometer (Shimadzu Corporation, Tokyo, Japan) was used to determine the surface plasmon resonance (SPR).

Surface-modified GMNs with PSS. A pure core/shell of $\mathrm{Fe}_{3} \mathrm{O}_{4} / \mathrm{Au}$ nanoparticle was obtained by dispersing GoldMag nanoflower $\left(\mathrm{Fe}_{3} \mathrm{O}_{4} / \mathrm{Au} / \mathrm{Fe}_{3} \mathrm{O}_{4}\right)$ with cationic surfactant, CTAB (22). After ultrasonic treatment at $45 \mathrm{~Hz}$ for $20 \mathrm{~min}$, $10 \mathrm{mg}$ nanoflower particles were gently mixed with $6 \mathrm{ml}$ CTAB (5 mmol/l) and sonicated for $40 \mathrm{~min}$. Subsequently, the CTAB-GMNs (GoldMag nanoparticles) were separated magnetically through the application of an external permanent magnet, and the supernatant (solution containing uncapped CTAB) was removed. A PSS solution $(6 \mathrm{ml}, 0.1 \mathrm{mg} / \mathrm{ml})$ was added to the CTAB-capped GMNs particles under sonication for $30 \mathrm{~min}$ and then left to stand for $2 \mathrm{~h}$. PSS-GMNs were magnetically separated and the supernatant was discarded; this step was repeated twice. Then, the PSS-GMNs were suspended in $7 \mathrm{ml}$ of deionized water.

Conjugation of PSS-GMNs with targeted moieties. PSS-GMNs $(1 \mathrm{mg})$ were equilibrated in the $600 \mu \mathrm{l}$ of phosphate buffer (1X PB, pH 7.2), containing $40 \mu \mathrm{g}$ of the targeted moiety (anti-digoxin or anti-FITC antibody). This mixture was shaken at $180 \mathrm{rpm}$ for $1 \mathrm{~h}$ at $22^{\circ} \mathrm{C}$. After $1 \mathrm{~h}$ of incubation, the unconjugated antibodies were removed by washing in a magnetic field. A blocking buffer (1X PB buffer, $\mathrm{pH} 7.2$, containing 3\% BSA and 5\% calf serum) was added to the conjugates, and the mixture was incubated for $1 \mathrm{~h}$. After incubation for $2 \mathrm{~h}$, anti-digoxin antibody or anti-FITC antibody functionalized PSS-GMNs conjugates, were magnetically separated and then suspended in buffer (1X PB, pH 7.2, containing $1 \% \mathrm{BSA}$ ) at $2-8^{\circ} \mathrm{C}$ prior to use. The conjugation efficiency was calculated by determining the concentration of anti-digoxin or anti-FITC antibody in the solution prior to and following coupling using the Lowry protein concentration assay, with BSA as a protein standard (23).

Preparation of LFA device. Streptavidin and goat anti-mouse $\mathrm{IgG}$ were printed on a porous nitrocellulose membrane to form the test line ( $\mathrm{T}$ line) and control line ( $\mathrm{C}$ line), respectively, using a HM3010 BioJet dispenser (BioDot, Inc., Irvine, CA, USA). To detect the genotype of each SNP, two complementary strips were run using half the volume of the same PCR product separately. One strip detects wild-type alleles (WT channel) and the other detects mutant alleles (M channel). The probe solution containing PGMNs with an anti-digoxin antibody or anti-FITC antibody was dispensed on the conjugate pad of WT channel and M channel of the LFA, respectively. These strips were placed in a card box and stored in a sealed aluminum foil bag with desiccant silica gel at room temperature. The strips remain stable for 12 months. 
PCR-GoldMag based-LFA for the visual detection of ApoE genotypes. According to the principle of ARMS-PCR (24), the reverse primers were designed as biotin-labeled common primers, and the forward primers are allele specific primers with the nucleotide at their 3 ' terminus corresponding to the SNP site. To detect the genotype of each sample, two complementary reactions (ApoE 158 tube and ApoE 112 tube) were run separately. The primers were combined in two reaction mixtures to yield predicted amplification products of $451 \mathrm{bp}$ (Reaction ApoE 158: ApoE 158C, ApoE 158T) and 588 bp (Reaction ApoE 112: ApoE 112T, ApoE 112C). Each PCR reaction was performed in a total volume of $50 \mu \mathrm{l}$ containing 10X PCR buffer (10 mM Tris $\left.\mathrm{HCl}, 1.5 \mathrm{mM} \mathrm{MgCl}_{2}\right), 2.5 \mu \mathrm{l}$ DMSO, $0.2 \mathrm{mM}$ dNTP mixture (dATP, dCTP, dGTP and dUTP), 0.5 U HotMaster Taq DNA Polymerase, $0.5 \mathrm{U}$ of UDG polymerase, $0.1 \mu \mathrm{M}$ common reverse primer and $4 \mu \mathrm{l}$ DNA template ( $4 \mu 1$ TE buffer without DNA served as a control in all experiments). The ApoE 158 reaction mixture also contained $0.05 \mu \mathrm{M}$ ApoE 158C and ApoE 158T forward primers. Similarly, the ApoE 112 reaction mixture contained $0.05 \mu \mathrm{M}$ ApoE $112 \mathrm{~T}$ and ApoE $112 \mathrm{C}$ forward primers. An Applied Biosystems 2720 PCR Thermal Cycler (Thermo Fisher Scientific, Inc.) was used for the amplification. The amplification was commenced with a UDG incubation step $\left(50^{\circ} \mathrm{C}\right.$ for $\left.2 \mathrm{~min}\right)$, initial denaturation/UDG inactivation step $\left(95^{\circ} \mathrm{C}\right.$ for $\left.5 \mathrm{~min}\right)$, followed by 30 cycles of $95^{\circ} \mathrm{C}$ for $30 \mathrm{sec}$ and $65^{\circ} \mathrm{C}$ for $1 \mathrm{~min}$, and a final extension at $65^{\circ} \mathrm{C}$ for $5 \mathrm{~min}$. Subsequent to PCR amplification, the whole reaction volume $(50 \mu \mathrm{l})$ was loaded on the sample pad of the WT channel and M channel of LFA strips and the results were analyzed.

The reference DNA samples with different ApoE genotypes, confirmed by direct sequencing (Sangon Biotech Co., Ltd., Shanghai, China), were used to validate the method. The 3 ' penultimate or antepenultimate nucleotide (underlined in Table I) was mismatched to enhance specificity of the assay (25). The sensitivity was evaluated by varying the concentrations of DNA samples.

Clinical application. Human whole blood samples $(n=305)$ were obtained from the Shaanxi Provincial People's Hospital (Xi'an, China). All subjects were self-reported to be from the Chinese Han populations and unrelated to each other. Informed written consent was obtained for participation in the present study, which was approved by the Human Subjects Ethical Committee of Northwest University. Genomic DNA was extracted from $200 \mu \mathrm{l}$ whole blood sample by Whole Blood Genomic DNA Isolation Kit from Xi'an GoldMag Nanobiotech Co., Ltd. (Xi'an, China) according to manufacturer's instructions. All DNA samples were tested to determine the genotypes of ApoE in a double-blind trial. Each sample was analyzed using PGMNs-based LFA strips and DNA sequencing (Sangon Biotech Co., Ltd.) as a comparison.

Statistical analysis. Statistical analyses were performed using Microsoft Excel version 14.0 (Microsoft Corporation, Redmond, WA, USA) and SPSS software version 16.0 (SPSS, Inc., Chicago, IL, USA). Statistical differences were determined using a Chi-squared test. $\mathrm{P}<0.05$ was considered to indicate a statistically significant difference.

\section{Results}

Preparation of GoldMag probes for LFA. GoldMag nanoparticles were synthesized and characterized as described previously (21). The general scheme to functionalize nanoflower GMNs with CTAB and PSS coating, is represented in Fig. 1A. The addition of cationic surfactant CTAB to the $\mathrm{Fe}_{3} \mathrm{O}_{4} / \mathrm{Au} / \mathrm{Fe}_{3} \mathrm{O}_{4}$ particles inhibited the absorption of additional $\mathrm{Fe}_{3} \mathrm{O}_{4}$ petals to the $\mathrm{Fe}_{3} \mathrm{O}_{4} / \mathrm{Au}$ core shell structure. CTAB stabilized the core/shell structure by neutralizing layers of surface positive charge to prevent aggregation. However, CTAB-coated particle dispersions are frequently destabilized in salt and buffer solution, resulting in partial aggregation and low recovery yields (26). In the present study, CTAB-capped coated $\mathrm{Fe}_{3} \mathrm{O}_{4} / \mathrm{Au}$ particles were stabilized by wrapping the CTAB layer with PSS (Fig. 1A).

The successful attachment of PSS was demonstrated in FT-IR spectra (Fig. 1B). The PSS spectrum has typical absorption features of neat polystyrene and sulfonate group $\left(\mathrm{SO}_{3}^{-}\right)(27,28)$, including a primary absorption of the stretching vibrations that correspond to asymmetric stretching of $-\mathrm{CH}_{2}-$ at 2,916 $\mathrm{cm}^{-1}, \mathrm{C}-\mathrm{C}$ stretching of $\mathrm{sp}^{2}$ hybridized carbon atoms at $1,597 \mathrm{~cm}^{-1}$, para-disubstituted benzenes $(\mathrm{C}=\mathrm{C})$ at $1,438 \mathrm{~cm}^{-1}$, symmetric vibrations of $-\mathrm{SO}_{3}$ groups in PSS chains at $1,158 \mathrm{~cm}^{-1}$ and stretching of hydroxyl groups of $-\mathrm{SO}_{2}-\mathrm{OH}$ over the region $3,700-3,000 \mathrm{~cm}^{-1}$. The spectra for CTAB-coated GMNs exhibited the distinctive absorption peaks centered at 2,920, 2,850, 1,401 , and $960 \mathrm{~cm}^{-1}$, which represented symmetric $(2,920$ and $\left.2,850 \mathrm{~cm}^{-1}\right)$ and asymmetric $\left(1,401 \mathrm{~cm}^{-1}\right)$ stretching of the $\mathrm{C}-\mathrm{H}$ bond in CTAB and quaternary amine $\left(960 \mathrm{~cm}^{-1}\right)$ stretching of CTAB (19), respectively. For PGMNs, the characteristic absorption bands of PSS were observed, whereas the distinctive peaks of CTAB were not detected. This result demonstrated the presence of the polymer on the particle, indicating that the PSS functionalized GMNs had been successfully prepared.

The stepwise conjugation of functional groups on the GMNs was then monitored by measuring the surface charges at different stages of synthesis. As presented in Fig. 1C, a positive zeta potential $(+27.5 \mathrm{mV})$ in neutral $\mathrm{pH}$ (water) of CTAB-capped GMNs, is attributed to the positive charge of the trimethyl ammonium group $\left[-\mathrm{N}\left(\mathrm{CH}_{3}\right)_{3}{ }^{+}\right]$of CTAB. Following the PSS coating of the nanoparticle surfaces, the zeta potential shifted to negative values $(-37.6 \mathrm{mV})$. This reversal of zeta potential upon the PSS coating indicates the presence of PSS on the nanoparticle surfaces, the PSS exhibits a constant negative charge above pH $2(18,29)$. Negative zeta potential formulations help repel each particle in the suspension, ensuring long-term stability and avoiding particle aggregation (30). When PGMNs were added into sodium chloride solution with an ionic strength of $30 \mathrm{mmol} / 1$ or $1 \mathrm{X} \mathrm{PB}$ buffer at $\mathrm{pH}$ 7.2, a slight decrease in zeta potential of PGMNs was observed due to lowering of ion screen effects of PSS in the electrolyte solution.

For assay development, PGMNs were conjugated to anti-digoxin antibodies or anti-FITC antibodies to construct GoldMag probes. Bioconjugation of particles with targeted antibodies was confirmed by dynamic light scattering measurement, SPR band analysis and the protein concentration assay. As demonstrated in Fig. 1D, the mean hydrodynamic diameter of particles increased from 68 to $95 \mathrm{~nm}$ (PGMNs-anti-digoxin) 
Table I. Nucleotide sequences of the primers used for ApoE genotyping.

Primer

ApoE 158C (wild-type) forward primer

ApoE 158T (mutant) forward primer

ApoE 112T (wild-type) forward primer

ApoE 112C (mutant) forward primer
Common reverse primer

Sequence

5'-digoxin-ATGCCGATGACCTGCAGACGC-3'
5'-FITC-ATGCCGATGACCTGCAGACGT-3'
5'-digoxin-CGCGGACATGGAGGACGTTT-3'
5'-FITC-CGCGGACATGGAGGACGTTC-3'
5'-biotin-GTTCAGTGATTGTCGCTGGGCA-3'

5'-digoxin-ATGCCGATGACCTGCAGACGC-3'

5'-FITC-ATGCCGATGACCTGCAGACGT-3'

5'-FITC-CGCGGACATGGAGGACGTTC-3'

The 3' penultimate or antepenultimate nucleotide (underlined) was mismatched to enhance specificity of the assay. ApoE, apolipoprotein E; FITC, fluorescein isothiocyanate.

A

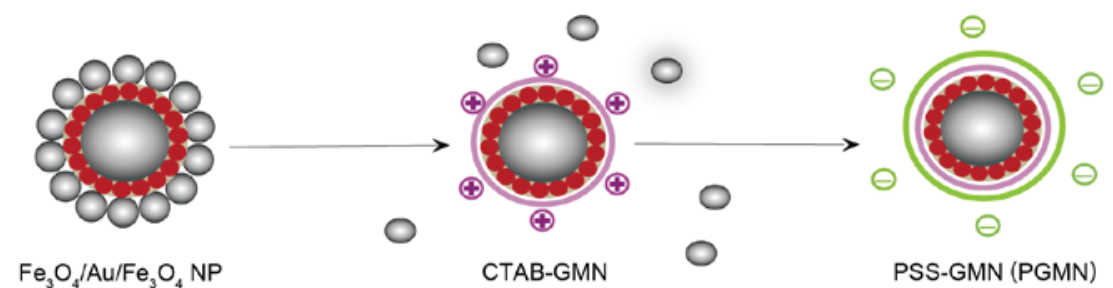

B

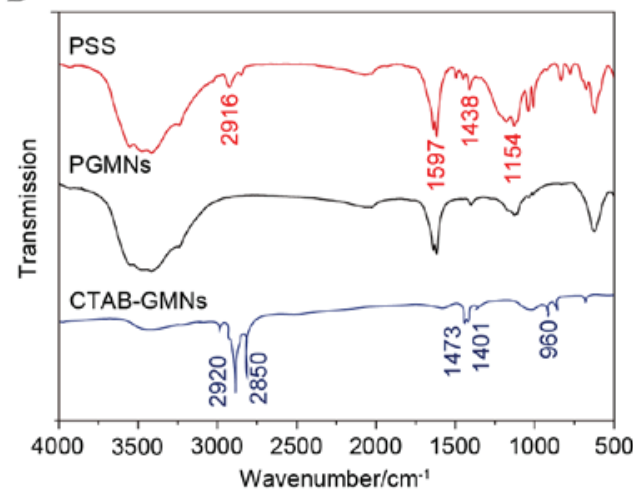

C

D

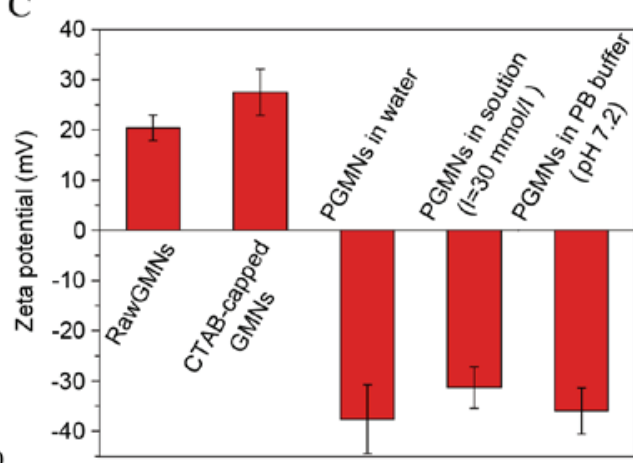

E

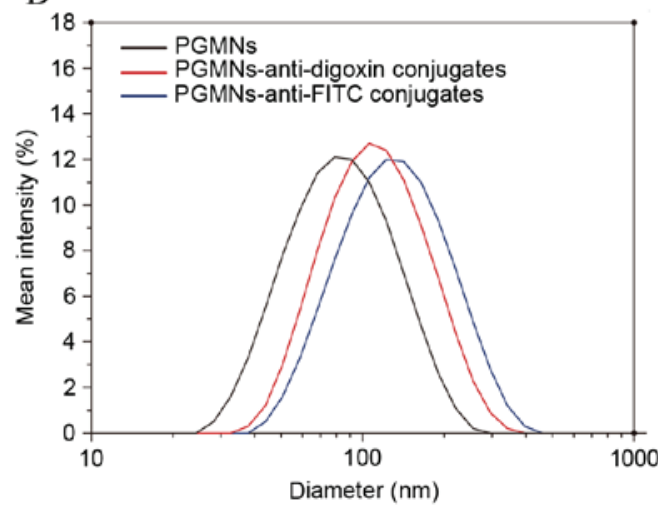

E

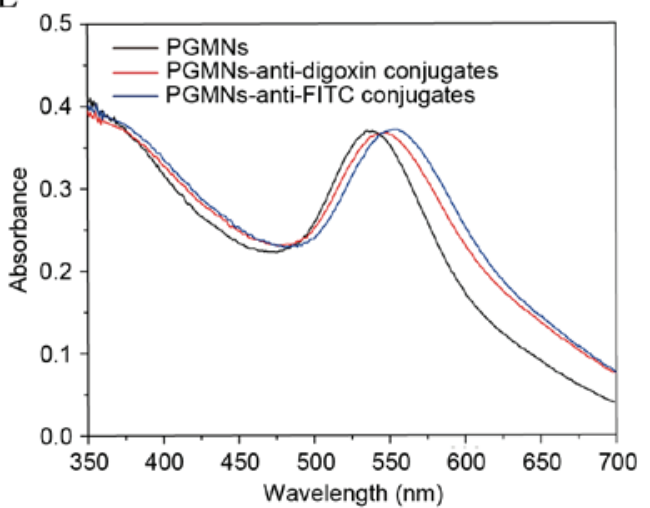

Figure 1. Generating PGMNs. (A) Schematic illustration of the process of surface modification, capping of the particles with surfactant CTAB and a follow-up coating of PSS. (B) FT-IR spectroscopy of CTAB-capped GMNs, pure PSS and PGMNs. (C) Zeta potential of GMNs, CTAB-capped GMNs and PGMNs suspended in water, PGMNs suspended in high electrolyte solution ( $\mathrm{I}=30 \mathrm{mmol} / \mathrm{l})$, and PGMNs suspended in PB. (D) Size distribution of PGMNs and PGMNs-antibody conjugates monitored by the dynamic light scattering analyzer. Reasonable increase of hydrodynamic size indicates the successful conjugation. (E) UV-vis spectrum of PGMNs-antibody conjugates revealed a corresponding red shift of surface plasmon resonance after PGMNs were conjugated with antibodies. NP, nanoparticle; CTAB, cetyltrimethylammonium bromide; GMN, GoldMag nanoparticle; PSS, polystyrenesulfonate; PGMN, polyelectrolyte-coated GMN; FITC, fluorescein isothiocyanate.

or $107 \mathrm{~nm}$ (PGMNs-anti-FITC) following conjugation. The increased hydrodynamic size following the conjugation suggested that antibodies were effectively coupled to the particles (31).
UV-vis data (Fig. 1E) indicated that antibody-labeled PGMNs exhibited a marked red shift compared with the unlabeled ones, from 538 to $547 \mathrm{~nm}$ (PGMNs-anti-digoxin) or $552 \mathrm{~nm}$ 
A

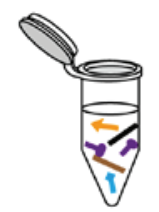

ApoE 158 or 112 tube

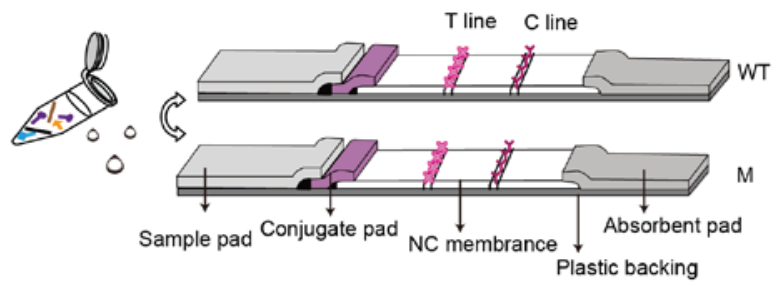

B

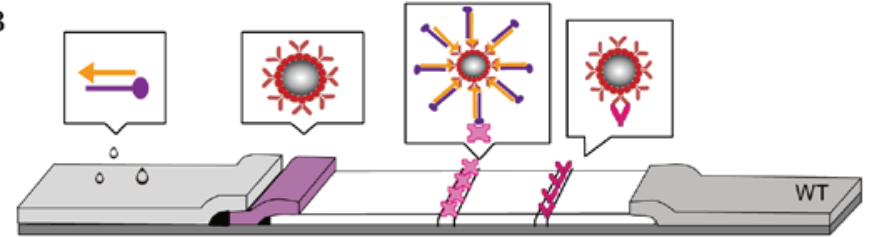

Forward (WT) prime

Fonward (M) primer

Reverse primer

Digoxin 4

FITC

Biotin

PCR target fragment

PGMNs-anti-digoxin 犄

antibody conjugates

PGMNs-anti-FITC

antibody conjugates 삿

Streptavidin 53

Goat anti-mouse IgG
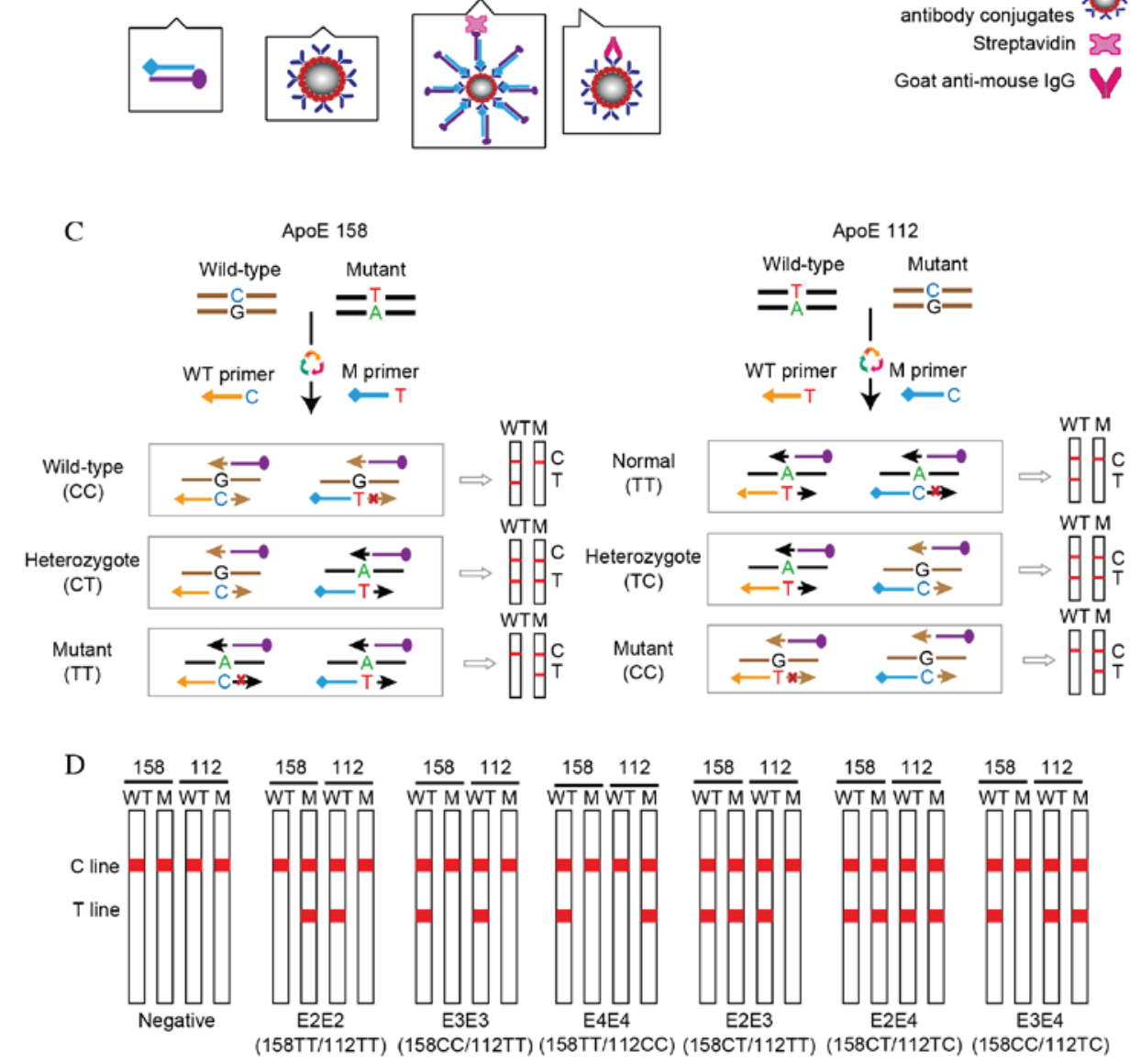

G

Figure 2. Schematic of the PCR-GoldMag LFA system. (A) Detecting one sample requires two reactions: ApoE 158 and ApoE 112. Detection of each PCR product using two complementary strips (WT channel and M channel). (B) Schematic diagram of the LFA strips based on the antibody-functionalized PGMN probes for visual detection of the PCR products. The conjugate pads of WT and M channel contain dispensed digoxin-conjugated PGMNs and FITC-conjugated PGMNs, respectively. The target fragments carrying the labels of digoxin/FITC and biotin are able to conjugate with PGMNs-anti-digoxin/FITC antibody complexes, which are captured by streptavidin on the test line with an appearance of a red band on the WT/M channel. The excessive PGMNs-antibody complex is precipitated by goat anti-mouse IgG on the control line. (C) Schematic illustration of the formation of digoxin/FITC-and biotin-conjugated duplex DNA complexes based on multiple amplification refractory mutation system-PCR. (D) Representative diagrams of LFAs in the presence of different ApoE phenotypes and negative control. LFA, lateral flow assay; ApoE, apolipoprotein E; T line, test line; C line, control line; NC, nitrocellulose; WT, wild-type channel; M, mutant channel; FITC, fluorescein isothiocyanate; PCR, polymerase chain reaction; PGMN, polyelectrolyte-coated GoldMag nanoparticle.

(PGMNs-anti-FITC) where the plasmon resonance band appears (32). This shift is likely to be caused by the surface chemistry change of the nanoparticles from PSS to antibody (33). The optimization of PGMNs-antibody conjugates was defined by the protein concentration assay as described in a previous report (23), and the appropriate amount of anti-digoxin or anti-FITC antibodies immobilized on the PGMNs was about 60 and $48 \mu \mathrm{g} / \mathrm{mg}$, respectively. 

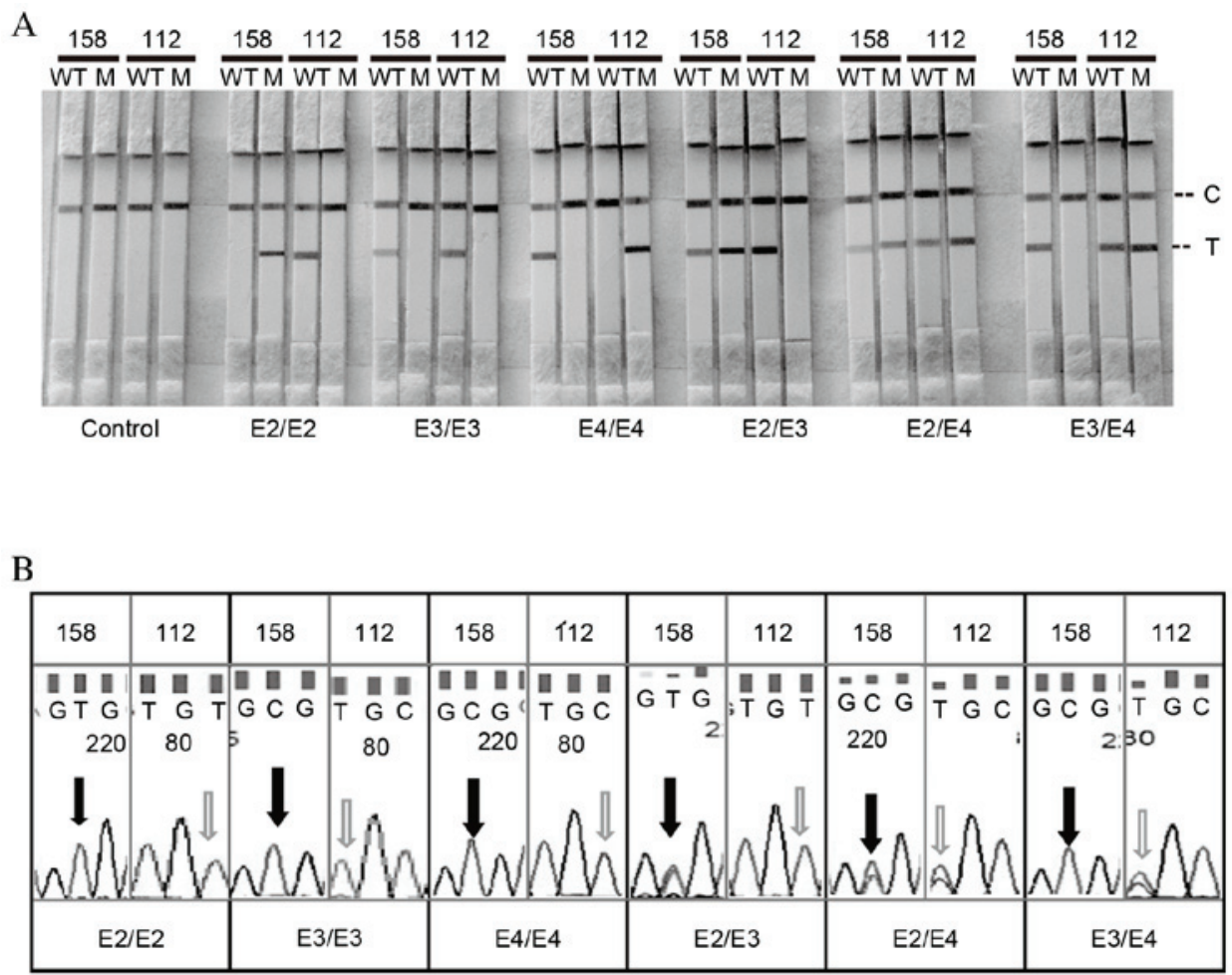

Figure 3. Specificity. Specificity test results of the amplification refractory mutation system method using $4 \mu 1$ each of DNA templates by (A) LFA and (B) direct DNA sequencing. LFA, lateral flow assay; 158, apolipoprotein E 158 polymorphism; 112, apolipoprotein E 112 polymorphism; WT, wild-type channel; M, mutant channel; C, control line; T, test line; E2, Cys158/Cys112 allele; E3, Cys158/Arg112 allele; E4, Arg158/Arg112 allele.

Principles of PCR-GoldMag LFA. The principle of visual detection of ApoE genotypes using PCR-GoldMag LFA system is illustrated in Fig. 2. The method consists of the following two steps (Fig. 2A): i) Amplification of one sample in two reactions, ApoE 158 and ApoE 112 tubes; and ii) PCR products of each tube are loaded onto the sample pads of two complementary LFA strips following amplification. One strip detects wild-type alleles (termed 'WT channel') and the other detects mutant alleles (termed ' $M$ channel'). The LFA strip is composed of five parts (Fig. 2B): A sample pad, a conjugate pad, a strip of nitrocellulose membrane, an absorbent pad and a plastic backing. Detection of each PCR product is performed using two complementary strips (WT and M channel) with conjugate pads pre-dispensed with PGMNs-anti-digoxin antibody conjugates and PGMNs-anti-FITC antibody conjugates, respectively.

To reduce the labor and cost, the multi-ARMS-PCR analysis was used to amplify wilt-type and mutant target SNP alleles in a single reaction tube, by simultaneously using allele-specific forward primers and a common, biotin-labeled reverse primer (Fig. 2C). Wild-type primers were labeled with digoxin and mutant primers were labeled with FITC. Genotyping of ApoE 158 and ApoE 112 was performed in two separate PCR tubes, with ApoE 158-specific primers to produce 158-specific amplicons (451 bp) and the other with ApoE 112-specific primers to produce 112-specific amplicons (588 bp). The PCR amplicons were synthesized with the 3 '-end of the primer complementary to the template. Thus, amplicons from the wild-type DNA were biotin and digoxin labeled, whereas the mutant amplicons were biotin and FITC labeled (Fig. 2C).
The visual detection of the amplicons was performed on the LFA strips. No purification of the PCR products was required prior to detection by LFA. In the test procedure, the PCR products migrate through the membrane strip. PCR target fragments, if labeled with digoxin, form a complex with the pre-fabricated PGMNs-anti-digoxin antibody conjugates on the adjacent conjugate pad of the WT channel strip. Similarly, PCR target fragments labeled with FITC form a complex with the pre-fabricated PGMNs-anti-FITC antibody conjugates of the $\mathrm{M}$ channel strip. The subsequent DNA-PGMNs-antibody conjugates migrate across the membrane strip until captured by pre-immobilized streptavidin on the $\mathrm{T}$ line forming the positive result of a red band. The red band at the $\mathrm{T}$ line of the WT channel strip indicates the presence of wild-type fragments, and the red band at $\mathrm{T}$ line of the $\mathrm{M}$ channel strip indicates presence of mutant fragments. The excess of PGMNs-antibody conjugates is captured at the $\mathrm{C}$ line by goat anti-mouse IgG that suggests the successful performance of the LFA system.

The final genotyping result of a sample for one locus requires visual inspection of the color development on the $\mathrm{T}$ lines of both the WT and M channel strips (Fig. 2C). For wild-type sample, a distinct red band is only visible on the T line of the WT channel, whereas the T line of the M channel does not have a color band. By contrast, for homozygous mutant samples, the red band appears exclusively on the $\mathrm{M}$ channel strip and not on the WT channel strip. However, when red bands with similar intensities are present on the $\mathrm{T}$ lines of WT and M strips, it indicates a heterozygous mutant sample. The detection of different ApoE phenotypes and negative control (no DNA) is illustrated in Fig. 2D. 
A

ApoE 158 reference DNA templates

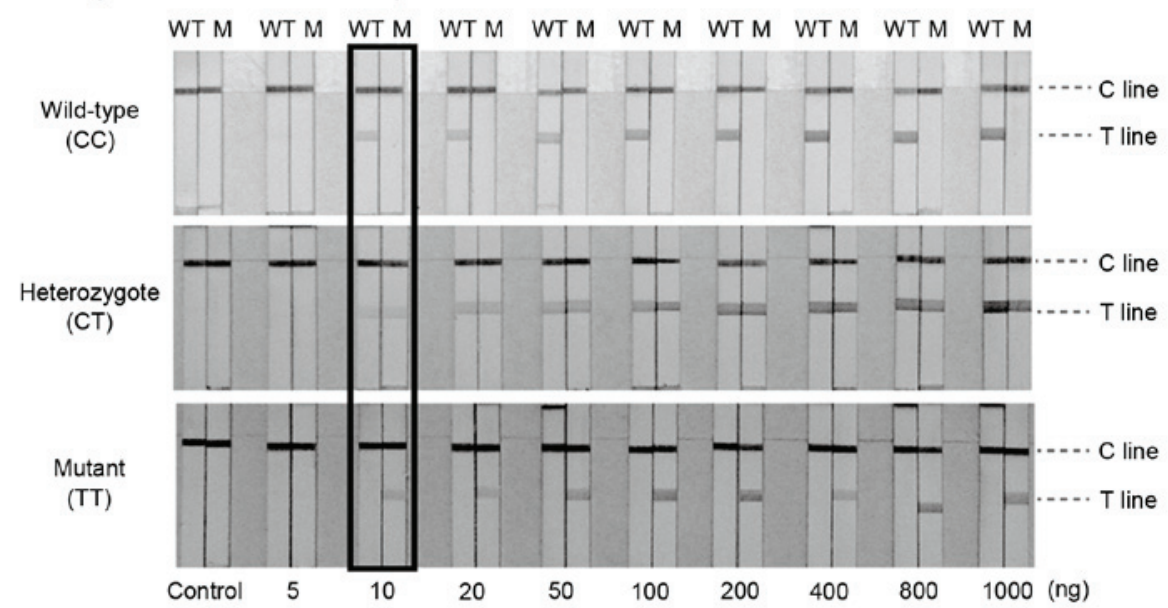

B ApoE 112 reference DNA templates

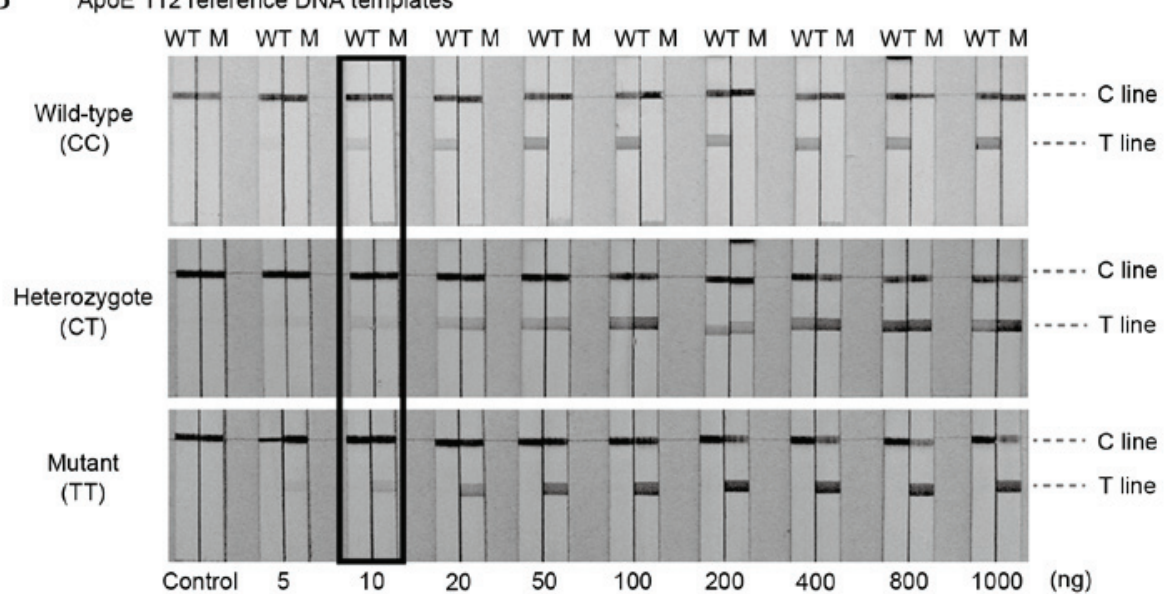

Figure 4. Sensitivity. Typical responses of LFAs with increasing quantities of (A) ApoE 158 and (B) ApoE 112 reference DNA templates. From left to right, the quantities of target DNA were 0,5,10, 20,50, 100, 200, 400, 800 and 1,000 ng. The minimum amount of target DNA for PCR that reacted with the particle conjugates causing coloration in the T-line is considered to be the detection limit of the assay, and the limit of detection of PCR-GoldMag LFA was as low as $10 \mathrm{ng}$. The limits of detection obtained with LFA are highlighted. LFA, lateral flow assay; ApoE, apolipoprotein E; 158, ApoE 158 polymorphism; 112, ApoE 112 polymorphism; WT, wild-type channel; M, mutant channel.

Performance of the LFA for ApoE genotyping. To obtain the optimal performance, potential variables, including ARMS, LFA preparation and detection conditions, were considered. All primers were designed to avoid cross-hybridization. In order to ensure the specificity of the primers, an additional mismatch at the penultimate or antepenultimate nucleotide of the 3 ' terminus of allele specific forward primers was introduced based on principle of ARMS-PCR (24). To increase the specificity and to reduce the PCR running-time, a combination of primer annealing and extension steps in the PCR was performed. By using a two-step PCR, the running-time was greatly reduced (30 cycles within $60 \mathrm{~min}$ ).

Specificity of the LFAs. The specificity of ApoE genotyping was an important consideration for the present study. For specificity analysis, six ApoE genotypes (E2/E2, E3/E3, $\mathrm{E} 4 / \mathrm{E} 4, \mathrm{E} 2 / \mathrm{E} 3, \mathrm{E} 2 / \mathrm{E} 4$, and E3/E4) were analyzed using the LFA system (Fig. 3A). In addition, ApoE genotyping results detected by DNA sequencing were performed as a comparison (Fig. 3B). The GoldMag-based LFA can clearly differentiate one base mutations between wild-type target DNA and mutant target DNA, allowing successfully distinction between the six ApoE genotypes.

Sensitivity of the LFAs. Under the optimum conditions, the sensitivity of LFA was evaluated by using various quantities of DNA samples with known ApoE genotypes. Different quantities of target DNA were used for sensitivity analysis $(5,10,20,50,100,200,400,800$ and 1,000 ng, and $0 \mathrm{ng}$ as a negative control). Fig. 4 presents the representative images of LFAs using different concentrations of ApoE 158 (Fig. 4A) and ApoE 112 (Fig. 4B) reference DNA templates. No red band was observed on the T line of the LFA in the absence of DNA (control). The signal intensity correspondingly increased as the target DNA concentration increased. The red bands on the $\mathrm{T}$ line were observed with $\geq 10 \mathrm{ng}$ of target DNA, which was considered to be the limit of detection of this assay.

Detection of clinical samples. To test the robustness of the proposed method for the ApoE genotypes, 305 clinical samples from a Han Chinese cohort were analyzed using the PCR-GoldMag LFA. In addition to the LFA, direct DNA 
Table II. Genotypes and frequency of ApoE variants in the Han Chinese cohort.

\begin{tabular}{lccc}
\hline \multirow{2}{*}{ Genotype } & $\begin{array}{c}\text { ApoE 158 variable } \\
\text { nucleotides }\end{array}$ & $\begin{array}{c}\text { ApoE 112 variable } \\
\text { nucleotides }\end{array}$ & $\begin{array}{c}\text { Frequency } \\
(\%)\end{array}$ \\
\hline E2/E2 & TT & TT & 0.95 \\
E3/E3 & CC & TT & 67.62 \\
E4/E4 & CC & CC & 1.90 \\
E2/E3 & CT & TT & 8.24 \\
E2/E4 & CT & TC & 3.81 \\
E3/E4 & CC & TC & 17.48 \\
\hline
\end{tabular}

n=305. ApoE, apoplipoprotein E; E2, Cys158/Cys112 allele; E3, Arg158/Cys112 allele; E4, Arg158/Arg112 allele.

sequencing was performed. All analyzed samples produced an unambiguous ApoE genotype. Of the 305 samples analyzed in the current study, the allele frequency of E2, E3 and E4 was $6.98,80.48$ and $12.54 \%$, respectively. The frequency of each of the ApoE genotypes is presented in Table II. All genotyping results $(100 \%)$ were in accordance with the results of direct DNA sequencing. In the subjects $(n=305)$, the overall allele distribution was similar to a previous study (34), with no significant difference in allele distribution (Chi-squared test, $\mathrm{P}=0.598$ ). These results indicate the reliability of using the PCR-GoldMag LFA method for ApoE genotyping.

\section{Discussion}

The present study demonstrated a PCR-GoldMag LFA for visual detection of ApoE genotypes using PSS-functionalized GoldMag nanoparticles as a carrier. To the best of our knowledge, this represents the first attempt to perform ApoE genotyping by integrating the multi-ARMS-PCR with LFA. GoldMag nanoparticles allow visual detection of the PCR product by observing the red color on an LFA strip. A prerequisite for immunoassay development of GMNs is sufficient functionalization, which maintains GMNs in a stable colloidal and monodispersed state with the ability to conjugate to targeted moieties. This was achieved in the present study with a fast and simple coating procedure using PSS. The detection capabilities of the PCR-GoldMag LFA system were examined using various quantities of DNA samples, with known ApoE genotypes. The system accurately assesses a broad detection range of initial starting genomic DNA quantity from $10 \mathrm{ng}$ to $1,000 \mathrm{ng}$, with the limit of detection reaching $10 \mathrm{ng}$. The specificity of the method was also confirmed using known ApoE genotypes, and no false positive results were observed. The performance of the LFA was further confirmed using 305 clinical samples and demonstrated to be a reliable method for determining the ApoE genotype. The entire protocol of the established method, including PCR and the LFA, can be performed in $1.5 \mathrm{~h}$. No purification of the PCR products or expensive detection instruments is required. The assay is also easy to use and does not require highly qualified trained personnel to be performed.
It has been previously proposed that the ApoE polymorphism may be associated with a high of developing certain diseases. The exposure of ApoE4 to contemporary environmental conditions (Western diet and longer lifespan) may have rendered it a susceptibility allele for coronary artery disease and Alzheimer's disease (35). The present study determined the frequency of the ApoE alleles, including the ApoE4 allele, in a cohort of northern Chinese Han population subjects.

The allelic frequencies of ApoE vary substantially around the world. Recently, a large study analyzed the ApoE allele distribution in China (34), including 19 separate cohorts, reporting distributions of 8.5, 83.0 and 8.5\% for the E2, E3 and $\mathrm{E} 4$ alleles, respectively $(\mathrm{n}=3,679)$. This particular previous study also found a conspicuous south-to-north gradient of ApoE4 frequencies in China, with the proportion of ApoE4 carriers at $4.9 \%$ in subjects from Kunming and increased to $17.5 \%$ subjects from Harbin (34). In the subjects of the current study ( $\mathrm{n}=305)$, the overall allele distribution was similar to the previous study, with no significant difference in allele distribution (Chi-squared test, $\mathrm{P}=0.598$ ).

In conclusion, the current study demonstrated that the PCR-GoldMag lateral flow assay is a simple, sensitive, rapid and cost-effective method for ApoE genotyping. This novel approach may be adapted for the detection of other important SNPs and be readily utilized for wide applications in molecular diagnosis laboratories and for point-of-care genotype analysis.

\section{Acknowledgements}

This study was supported by the Project of National Great New Drug Research and Development China (grant no. 2012ZX09506001-001, YC), the National Natural Science Foundation of China (grant no. 31200749) and the National Institute of Health (grant no. P20RR016457 from the INBRE Program of the National Center for Research Resources, YW).

\section{References}

1. Hirschhorn JN and Daly MJ: Genome-wide association studies for common diseases and complex traits. Nat Rev Genet 6: 95-108, 2005.

2. Shabo A: Integrating genomics into clinical practice: Standards and regulatory challenges. Curr Opin Mol Ther 10: 267-272, 2008.

3. Bertram L and Tanzi RE: Thirty years of Alzheimer's disease genetics: The implications of systematic meta-analyses. Nat Rev Neurosci 9: 768-778, 2008.

4. Liu CC, Kanekiyo T, Xu H and Bu G: Apolipoprotein E and Alzheimer disease: Risk, mechanisms and therapy. Nat Rev Neurol 9: 106-118, 2013.

5. Lahiri DK, Sambamurti K and Bennett DA: Apolipoprotein gene and its interaction with the environmentally driven risk factors: Molecular, genetic and epidemiological studies of Alzheimer's disease. Neurobiol Aging 25: 651-660, 2004.

6. Hackler R, Schäfer JR, Motzny S, Brand S, Kleine TO, Kaffarnik H and Steinmetz A: Rapid determination of apolipoprotein E phenotypes from whole plasma by automated isoelectric focusing using PhastSystem and immunofixation. J Lipid Res 35: 153-158, 1994.

7. Nishimura M, Satoh M, Nishimura S, Kakinuma S, Sato K, Sawai S, Tsuchida S, Kazama T, Matsushita K, Kado S, et al: Human apolipoprotein e resequencing by proteomic analysis and its application to serotyping. PloS One 9: e85356, 2014.

8. Wu YY, Delgado R, Costello R, Sunderland T, Dukoff R and Csako G: Quantitative assessment of apolipoprotein E genotypes by image analysis of PCR-RFLP fragments. Clin Chim Acta 293: 213-221, 2000. 
9. Yi L, Wu T, Luo W, Zhou W and Wu J: A non-invasive, rapid method to genotype late-onset Alzheimer's disease-related apolipoprotein E gene polymorphisms. Neural Regen Res 9: 69-75, 2014.

10. Ghebranious N, Ivacic L, Mallum J and Dokken C: Detection of ApoE E2, E3 and E4 alleles using MALDI-TOF mass spectrometry and the homogeneous mass-extend technology. Nucleic Acids Res 33: e149, 2005

11. Donohoe GG, Salomäki A, Lehtimaki T, Pulkki K and Kairisto V: Rapid identification of apolipoprotein E genotypes by multiplex amplification refractory mutation system PCR and capillary gel electrophoresis. Clinical Chem 45: 143-146, 1999.

12. Poli M, Gatta LB, Dominici R, Lovati C, Mariani C, Albertini A and Finazzi D: Apolipoprotein E haplotyping by denaturing high-performance liquid chromatography. Clin Chem Lab Med 43: 512-518, 2005.

13. Koch W, Ehrenhaft A, Griesser K, Pfeufer A, Müller J, Schömig A and Kastrati A: TaqMan systems for genotyping of disease-related polymorphisms present in the gene encoding apolipoprotein E. Clin Chem Lab Med 40: 1123-1131, 2002.

14. Ben-Avi L, Durst R, Shpitzen S, Leitersdorf E and Meiner V: Apolipoprotein E genotyping: Accurate, simple, high throughput method using ABI Prism SNaPshot Multiplex System. J Alzheimers Dis 6: 497-501, 2004.

15. Lie P, Liu J, Fang Z, Dun B and Zeng L: A lateral flow biosensor for detection of nucleic acids with high sensitivity and selectivity. Chem Commun (Camb) 48: 236-238, 2012.

16. Wei Q, Xiang Z, He J, Wang G, Li H, Qian Z and Yang M: Dumbbell-like Au-Fe3O4 nanoparticles as label for the preparation of electrochemical immunosensors. Biosens Bioelectron 26: 627-631, 2010

17. Zhang C, Shen G, Shen Y and Zhang X: The development of an electrochemical immunosensor using a thiol aromatic aldehyde and PAMAM-functionalized Fe3O4@Au nanoparticles. Anal Biochem 485: 66-71, 2015.

18. Mehtala JG, Zemlyanov DY, Max JP, Kadasala N, Zhao S and Wei A: Citrate-stabilized gold nanorods. Langmuir 30: 13727-13730, 2014.

19. Yang D, Ma JZ, Zhang QL, Li NN, Yang J, Raju PA, Peng ML, Luo YL, Hui WL, Chen C and Cui Y: Polyelectrolyte-coated gold magnetic nanoparticles for immunoassay development: Toward point of care diagnostics for syphilis screening. Anal Chem 85: 6688-6695, 2013.

20. Hui W, Zhang S, Zhang C, Wan Y, Zhu J, Zhao G, Wu S, Xi D, Zhang Q, Li N and Cui Y: A novel lateral flow assay based on GoldMag nanoparticles and its clinical applications for genotyping of MTHFR C677T polymorphisms. Nanoscale 8 : 3579-3587, 2016.

21. Hui W, Shi F, Yan K, Peng M, Cheng X, Luo Y, Chen X, Roy V, Cui Y and Wang Z: Fe3O4/Au/Fe3O4 nanoflowers exhibiting tunable saturation magnetization and enhanced bioconjugation. Nanoscale 4: 747-751, 2012
22. Shi F, Hui W, Chen C and Cui Y: Surface modification and characterization of $\mathrm{Fe}_{3} \mathrm{O}_{4} / \mathrm{Au}$ composite nanoparticles. NANO 6: 145-151, 2011

23. Yang D, Ma J, Peng M, Zhang Q, Luo Y, Hui W, Jin T and Cui Y: Building nanoSPR biosensor systems based on gold magnetic composite nanoparticles. J Nanosci Nanotechnol 13: 5485-5492, 2013.

24. Little S: Amplification-refractory mutation system (ARMS) analysis of point mutations. Curr Protoc Hum Genet: Chapter 9:Unit 9.8, 2001. doi: 10.1002/0471142905.hg0908s07.

25. Liu J, Huang S, Sun M, Liu S, Liu Y, Wang W, Zhang X, Wang $\mathrm{H}$ and Hua W: An improved allele-specific PCR primer design method for SNP marker analysis and its application. Plant Methods 8: 34, 2012.

26. Rostro-Kohanloo BC, Bickford LR, Payne CM, Day ES, Anderson LJ, Zhong M, Lee S, Mayer KM, Zal T, Adam L and Dinney CP: The stabilization and targeting of surfactant-synthesized gold nanorods. Nanotechnology 20: 434005, 2009

27. Lee HY, Rwei SP, Wang L and Chen PH: Preparation and characterization of core-shell polyaniline-polystyrene sulfonate@ $\mathrm{Fe}_{3} \mathrm{O}_{4}$ nanoparticles. Materials Chemistry and Physics 112: 805-809, 2008.

28. Zalakain I, Politakos N, Ramos JA, Saralegi A, Etxeberria H, Mondragon I, Corcuera A and Eceiza A: Chemical and morphological characterization of sulfonated polystyrene brushes in different environments. Eur Polym J 49: 2120-2127, 2013.

29. Dorris A, Rucareanu S, Reven L, Barrett CJ and Lennox RB: Preparation and characterization of polyelectrolyte-coated gold nanoparticles. Langmuir 24: 2532-2538, 2008.

30. Billotey C, Wilhelm C, Devaud M, Bacri JC, Bittoun J and Gazeau F: Cell internalization of anionic maghemite nanoparticles: Quantitative effect on magnetic resonance imaging. Magn Reson Med 49: 646-654, 2003.

31. Ahmadi A, Shirazi H, Pourbagher N and Omidfar K: Synthesis and characterization of core-shell Au Fe oxide nanocomposites and their application for detecting immunological interaction. Monoclon Antib Immunodiagn Immunother 33: 74-79, 2014.

32. McFarland AD, Haynes CL, Mirkin CA, Van Duyne RP and Godwin HA: Color my nanoworld. J Chem Educ 81: 544A, 2004.

33. Kaur K and Forrest JA: Influence of particle size on the binding activity of proteins adsorbed onto gold nanoparticles. Langmuir 28: 2736-2744, 2012.

34. Hu P, Qin YH, Jing CX, Lu L, Hu B and Du PF: Does the geographical gradient of ApoE4 allele exist in China? A systemic comparison among multiple Chinese populations. Mol Biol Rep 38: 489-494, 2011.

35. Corbo RM and Scacchi R: Apolipoprotein E (APOE) allele distribution in the world. Is APOE" 4 a 'thrifty' allele? Ann Hum Genet 63: 301-310, 1999. 